\title{
Können Erzählungen lügen?
}

Abstract: Narrative explanations of events occur in two forms, causal and configurative. Causal explanations claim a referential truth. The truth of a complex causal explanation results from the conjunction and weighting of its parts. In contrast, a configurative explanation of events aims at the overall narrative structure. Schemes of action or plot types assign meanings to an event which cannot be true in a referential sense. Rather, the claim to validity of a configurative explanation is subject to criteria such as conciseness, meaningfulness, or persuasiveness. The supposedly inevitable rhetorical character of narratives only limits the truth claim of one form of narrative explanation, namely the configurative one. Causal narrative explanations, on the other hand, are no more, but also no less truthful than empirical statements about our reality in general. Configurative explanations strive for suggestion, causal explanations for explication.

In der öffentlichen Debatte über ,alternative facts‘ und postfaktisches Erzählen wird die Erzählung gern als erkenntnistheoretisch, politisch und moralisch problematischer Gegenbegriff zu soliden Fakten und Beweisen verwendet. Erzählungen als solche, scheint es, sind nicht wahrheitsfähig, sondern stets rhetorische Instrumente im Dienst manipulativer Absichten. Sie wären dann nicht nach ihrer Wahrheit oder Falschheit zu beurteilen, sondern nach ihrer Überzeugungskraft und allenfalls noch nach ihrer politischen oder moralischen Legitimität. So schreiben beispielsweise Vincent Hendricks und Mads Vestergaard in Verlorene Wirklichkeit? An der Schwelle zur postfaktischen Demokratie: „Eine Demokratie befindet sich in einem postfaktischen Zustand, wenn nicht länger Tatsachen und Beweise, sondern opportune Narrative als Grundlage der Meinungsbildung in der öffentlichen Debatte und der Politik dienen“ (Hendricks und Vestergaard 2017, 5; meine Hervorhebungen, M. M.). Im postfaktischen demokratischen Diskurs seien „Fakten und Belege von zweitrangiger Bedeutung [...], da sie, verglichen mit unsachlichen Narrativen, nicht genügend Aufmerksamkeit erhalten“. „Auf diese Weise [dominieren] Narrative statt Tatsachen die öffentliche Agenda, Meinungen statt Kenntnisse, Stereotype statt repräsentative Beispiele, politische Blasen statt

Matías Martínez, Bergische Universität Wuppertal, martinez@uni-wuppertal.de əopen Access. (C 2021 Matías Martínez, publiziert von De Gruyter. (c) BY Dieses Werk ist lizenziert unter der Creative Commons Attribution 4.0 International Lizenz.

https://doi.org/10.1515/9783110693065-002 
politische Substanz“ (Hendricks und Vestergaard 2017, 7; meine Hervorhebungen, M. M.). Für Hendricks und Vestergaard sind „Narrative“ also Manipulationsinstrumente im Dienst der postfaktischen Leugnung von Tatsachen und Wahrheit: Der Bezug auf „Narrative statt Tatsachen“ untergrabe eine rationale politische Diskussion.

Der Begriff des ,Narrativs ${ }^{61}$ wird in der aktuellen Debatte allerdings nicht nur pejorativ gebraucht. Matthew D'Ancona etwa schreibt in Post Truth. The new war on truth and how to fight back Erzählungen („narratives“) eine große Bedeutung für das „Post-Truth“-Zeitalter zu (,central relevance to the Post-Truth era“, D’Ancona 2017, 130). So wie Hendricks und Vestergaard meint auch D’Ancona, dass Erzählungen durchaus im Dienste manipulativer Absichten stehen könnten: „Trump's [...] success has been built upon a story as powerful as it is simple: that he can ,Make America Great Again““ (D’Ancona 2017, 131). Narrative könnten aber auch als Gegenmittel gegen Manipulationen dienen:

[L]isting the lies that Trump tells is hugely important, but it isn't enough [...]. To defend the truth against the President and those who will follow his lead, powerful counter-narratives are required. [...] The task for those who do not share the politics of Trump or the Brexiteers is to speak with empathy and candour, to wrap facts in stories that speak to ordinary human concerns. Narrative must never violate or embellish truth; it should be its most powerful vehicle. (D’Ancona 2017, 131 und 136; meine Hervorhebungen, M. M.)

Anders als Hendricks und Vestergaard sieht D’Ancona Erzählungen also nicht grundsätzlich als schädlich an. Es scheint eher darauf anzukommen, ob sie im Dienste der richtigen oder der falschen Seite verwendet werden. Als „counternarratives“ könnten sie gegen Lügen wirken. Ungeachtet dieses Unterschieds sind sich Hendricks / Vestergaard und D’Ancona aber mit vielen anderen grundsätzlich darin einig, dass Erzählungen als solche nicht wahrheitsfähig seien. Vielmehr erscheinen sie ihnen als rhetorische Instrumente, um bestimmte Meinungen über strittige Sachverhalte hervorzurufen und erwünschte Haltungen zu insinuieren. Erzählungen (oder Narrative) besäßen dann keinen eigenen Wahrheitsgehalt. Folgt man dieser Auffassung, dann läge die Überzeugungskraft von Erzählungen paradoxerweise in ihrem nur scheinbaren Anspruch auf Wahrheit:

1 Im aktuellen Gebrauch werden die Substantive ,Narrativ‘, ,Narration‘ und ,Erzählung‘ weitgehend synonym verwendet (als beliebiger Beleg dafür siehe Biegon und Nullmeier 2014, 39). Der substantivische Anglizismus ,Narrativ‘ (zu engl. ,narrative`) verwischt allerdings die Unterschiede zwischen ,Erzählung‘ i. e. S. (als Geschehensdarstellung), ,Handlung‘ (als dargestelltes Geschehen) und ,Handlungsschema‘ (als typische Handlungs- und Deutungsstruktur einer bestimmten Gruppe von Erzählungen). 
Es bestünde ein Verblendungszusammenhang zwischen illusorischem Wahrheitsanspruch und realer Überzeugungskraft von Erzählungen.

Einer solchen Auffassung vom angeblich unvermeidlichen und unhintergehbaren Manipulationscharakter von Geschichten widerspricht nun aber die Praxis des Erzählens in zahlreichen sozialen Feldern, in denen wahrheitsheischende ,Wirklichkeitserzählungen' eine konstitutive Rolle spielen (siehe dazu ausführlich Klein und Martínez 2009). Schon in alltäglichen Erzählsituationen ist die Wahrheitsverpflichtung des Erzählers in der Regel eine selbstverständliche kommunikative Voraussetzung - was besonders dann deutlich wird, wenn diese Verpflichtung in Täuschungsabsicht verletzt wird. Aber auch hochgradig institutionalisierte Erzählumgebungen wie die Rechtsprechung setzen voraus, dass das kommunikative Format ,Erzählen' wahrheitsfähig ist, wenn Zeugenaussagen vor Gericht gehört werden - so sehr dieser Anspruch in jedem Einzelfall auch strittig sein mag (vgl. Volbert 2017).

Diese hier nur ansatzweise skizzierte Sachlage zeigt, dass widersprüchliche Auffassungen über den Wahrheitsanspruch von Erzählungen zirkulieren. Ich möchte im Folgenden versuchen, diese Widersprüche zu entwirren durch eine Unterscheidung zwischen zwei Arten narrativer Evidenz, die einerseits aus kausalen, andererseits aus konfigurativen Ereignisverknüpfungen entstehen. In bestimmter Hinsicht sind Erzählungen grundsätzlich wahrheitsfähig. Die oben skizzierte Auffassung, Erzählungen stünden stets im Gegensatz zu Fakten, unterstellt, Fakten seien objektivierbar, während Erzählungen solche Fakten unvermeidlich in ein arbiträres, nach rein rhetorischen Zielen gewähltes und bestimmtes temporales Verlaufs- und Deutungsschema überführten. Doch objektive Erkenntnis ist auch über Zeitverläufe und Ereignisketten möglich - diese sind sogar besonders relevante Gegenstände wissenschaftlichen, aber auch alltäglichen Wissens. Es gibt ein spezifisch narratives Wissen, das Geschehen in Form von wahrheitsfähigen Erzählungen erklärt.

Bevor ich diese These erläutere, ist noch eine Einschränkung vorzunehmen. Im Folgenden geht es nicht um die Frage, ob und inwiefern literarisch-fiktionale Erzählungen oder auch Werke, die sich in den Grauzonen zwischen fiktionalen und faktualen Texten bewegen, lügen können, sondern um sogenannte,faktuale‘ Erzählungen. Wer eine faktuale Erzählung vorbringt, schließt mit seinen Hörern oder Lesern stillschweigend einen Pakt über den konkreten Referentialitätsanspruch des Gesagten. Deshalb ist im Übrigen auch die Titelfrage, ob Erzählungen lügen können, verkürzt formuliert. $\mathrm{Zu}$ lügen setzt die kommunikative Absicht eines Sprechers voraus, seinen Adressaten zu täuschen, und besteht aus einem entsprechenden kommunikativen Akt, bei dem das narrative Kommunikat (in der Regel eine tatsachenwidrige ,Geschichte`) nur einen Teil der übergreifenden 
Kommunikationssituation bildet. Situationsenthobene Erzählungen allein können nicht lügen, Geschichten als solche nicht wahr oder falsch sein, solange sie nicht in die konkrete Pragmatik einer gegebenen Erzählsituation eingebettet sind.

\section{Erzählungen als kausale und konfigurative Erklärungen}

Worin besteht nun der oben postulierte Unterschied zwischen kausaler und konfigurativer Ereignisverknüpfung, und welche Folgen hat dieser für den Wahrheitswert von Erzählungen? Erzählungen können referentiell wahrheitsfähig sein und zu objektivem Wissen führen, wenn sie komplexe Ereignisse kausal erklären. Indem wir die Vorgeschichte einer Situation erzählen, erklären wir, wie und warum sie zustande gekommen ist. Damit verstehen wir die Situation als Ergebnis einer nicht nur chronologischen, sondern kausal strukturierten Ereignisfolge. In diesem Sinne bestimmt Arthur C. Danto bekanntlich eine Geschichte (,story') als eine zeitliche Folge von zwei zueinander kontradiktorischen Zuständen eines konstanten Geschehensträgers (vgl. Danto 1985, bes. 233-256): ,Max war hungrig, dann war er satt‘. Erzählungen liefern narrative Erklärungen (,narrative explanations`) für solche Zustandswechsel, indem sie ein Ereignis angeben, das den späteren Zustand verursacht: ,Max war hungrig, dann aß er Spaghetti und war (deswegen) satt‘: Die narrative Kohärenz einer Geschichte liegt dann darin, dass ihr Anfangs- und ihr Endzustand in einem kausalen Erklärungszusammenhang zusammengefasst werden. Erzählen ist für Danto also nicht nur eine Form der Geschehensdarstellung, sondern leistet auch eine kausale Erklärung des dargestellten Geschehens. Zeitlich aufeinander folgende Zustände werden zu einer Geschichte integriert, wenn der Unterschied zwischen früheren und späteren Zuständen nicht als ein bloßer Wechsel, sondern als eine aufgrund von Regeln erklärbare Veränderung aufgefasst wird. Die kausale Motivation integriert die Ereignisse in einen Erklärungszusammenhang. Die Ereignisse werden dann so verstanden, dass sie nicht grundlos, wie aus dem Nichts, aufeinander, sondern nach Regeln oder Gesetzen auseinander folgen. Erst mit Hilfe des erklärenden Zwischengliedes lässt sich die zeitliche Folge separater Zustände als eine Veränderung oder Entwicklung erkennen.

Nun sind Erzählungen natürlich in aller Regel komplexer als diese Minimalstruktur einer ,story“ im Sinne Dantos (vgl. Martínez 2017). Danto selbst unterscheidet deshalb zwischen ,atomarer' und ,molekularer' Erzählung (,atomic‘ und 
,molecular narrative'). Die atomare Erzählung weist die oben beschriebene dreiteilige Struktur auf, während die molekulare (oder auch ,genetische') mehrere atomare Einheiten aneinanderreiht. Doch auch das Konzept der molekularen Erzählung hält noch an einer unilinearen Ursache-Wirkungskette fest und kann deshalb die komplexen Kausalgefüge von Geschichten nicht angemessen erfassen. Erzählungen stellen kaum jemals einfache Ursache-Wirkungsketten dar, in der ein bestimmtes früheres Ereignis ein späteres notwendig und hinreichend determiniert. In der Regel präsentieren sie, wie John L. Mackie gezeigt hat, Gemengelagen aus intentionalen Handlungen und nicht-intendierten Geschehnissen, die insgesamt nicht monokausal, sondern in Form eines ,kausalen Feldes“ (,causal field‘) miteinander verflochten sind (vgl. Mackie 2002). Erzählte Ereignisse sind zumeist unterdeterminiert: Ereignis a trägt kausal zu einem späteren Ereignis b bei, aber determiniert es nicht. Geschichten sind kontingent, sie hätten auch anders verlaufen können.

Konzepte einer narrativen Erklärung wie ,story‘, ,molecular narrative‘ oder ,causal field' bleiben jedenfalls allesamt der Idee verpflichtet, dass die Erklärung eines Zustands aus der Erläuterung seiner kausalen Vorgeschichte besteht. Eine Erzählung erklärt ein Ereignis oder einen Zustand, indem sie die kausale Gemengelage von Geschehnissen darstellt, die diese hervorgebracht hat.

Annahmen über Typisierungen und Gesetze spielen dabei eine wichtige Rolle. Damit ein Ereignis als Erklärung für eine Veränderung dienen kann, muss es einem Ereignistyp zugeordnet werden, von dem man annimmt, dass er in der Regel aus ähnlichen Antezedentien ähnliche Folgen hervorbringt. Narrative Erklärungen subsumieren also den erzählten Einzelfall explizit oder implizit unter allgemeine, gesetzesartige Regeln. Das erinnert an Erklärungen nach dem von Carl Hempel und anderen aufgestellten sogenannten DN-Modell (zum Überblick siehe Henning 1980 und Lorenz 1997). Der wichtigste Unterschied zwischen narrativen und deduktiv-nomologischen Erklärungen liegt darin, dass narrative Erklärungen komplexe Erklärungsketten bilden, die zwar in ihren einzelnen Gliedern, nicht aber in ihrer Gesamtheit aus Gesetzen oder gesetzesartigen Regeln abgeleitet werden können.

Betrachten wir beispielsweise den Absturz des Passagierflugzeuges Boeing 737 Max der indonesischen Fluggesellschaft Lion Air am 29. Oktober 2018 nahe Djakarta, bei dem alle 189 Insassen starben. Der offizielle, 318 Seiten umfassende Abschlussbericht der nationalen indonesischen Luftsicherheitsbehörde KNKT stellte fest, dass viele Faktoren beim Absturz zusammenwirkten. $\mathrm{Zu}$ diesen Faktoren gehörten unter anderem,

- dass das automatische Steuerungsprogramm nicht auszuschalten war,

- dass ein Außensensor fehlerhaft war, 
- dass die Verarbeitung der Sensordaten vom Hersteller Boeing nicht richtig eingestellt war,

- dass Sensor und Software von der Flugfirma Lion Air nicht überprüft wurden,

- dass das Handbuch unvollständig war,

- dass das Pilotentraining mangelhaft war,

- dass die Kommunikation der Piloten mit den Fluglotsen fehlerhaft war.

Diese komplexe „Verkettung von Fehlern“ habe, so zusammenfassend ein Artikel der Frankfurter Allgemeinen Zeitung, zum Absturz geführt. Der Zeitungsartikel resümiert: „Wenn einer von ihnen nicht geschehen wäre, hätte es möglicherweise keinen Absturz gegeben“ (N. N. 2019). Statt von einer linearen „Verkettung“ von Ursachen und Wirkungen sollte man allerdings besser von einer komplexen Gemengelage unterschiedlich relevanter Faktoren sprechen. Der Kommissionsbericht der KNKT listet nicht weniger als 89 Faktoren auf (vgl. Komite Nasional 2019, 204-214) und unterscheidet bei seinen „findings“ zwischen verschiedenen Typen von Faktoren, nämlich „,conditions“, „events“ und „,circumstances“:

Findings are statements of all significant conditions, events or circumstances in the accident sequence. The findings are significant steps in the accident sequence, but they are not always causal, or indicate deficiencies. Some findings point out the conditions that preexisted the accident sequence, but they are usually essential to the understanding of the occurrence, usually in chronological order. (Komite Nasional 2019, 204)

Außerdem listet der Report neun zusätzliche „contributing factors“ auf, nämlich

actions, omissions, events, conditions, or a combination thereof, which, if eliminated, avoided or absent, would have reduced the probability of the accident or incident occurring, or mitigated the severity of the consequences of the accident or incident. (Komite Nasional 2019, 215)

Es wäre also extrem verkürzend, von ,der‘ Ursache für den Flugzeugabsturz zu sprechen. Gleichwohl können die Faktoren gewichtet werden. Zumindest die Fluggesellschaft Lion Air kam jedenfalls zu dem Schluss, dass die Hauptursache (,primary cause') für den Absturz das neu entwickelte, fehlerhafte Steuerungsprogramm Maneuvering Characteristics Augmentation System (MCAS) des Herstellers Boeing war: „The information contained by the KNKT during its investigation of the Flight JT610 Accident demonstrates that the primary cause of the Flight JT610 accident was Boeing's flawed design and development of the MCAS“ (Komite Nasional 2019, 318). Boeing widersprach zunächst diesem Befund und 
verwies auf die Komplexität der Sachlage. Nachdem es aber in den folgenden Monaten zu weiteren Unfällen und Abstürzen mit demselben MCAS-System kam, zog Boeing die damit ausgestatteten Jumbos aus dem Verkehr.

Anders als in diesem sehr detailliert untersuchten Fall sind narrative Erklärungen, wie sie im Alltag und in der Politik gegeben werden, in der Regel unvollkommen, nämlich partiell (das Explanans erklärt nicht alle, sondern nur einige Aspekte des Explanandum-Phänomens), skizzenhaft (relevante Antezedensdaten und Gesetze werden nur teilweise genannt), kontextbezogen (welche Faktoren genannt werden, hängt vom pragmatischen Kontext ab) und intuitiv (narrative Erklärungen beziehen ihre Plausibilität nicht aus dem expliziten Zugriff auf wissenschaftliche Theorien, sondern aus der impliziten Evidenz von common sense und tacit knowledge) (vgl. Haussmann 1996, 55-59). Diese vermeintlichen Defizite unterscheiden alltägliche narrative Kausalerklärungen aber nur graduell von detaillierteren Untersuchungen wie beim Absturz der Lion Air-Boeing 737 Max. Sie annullieren aber nicht ihren epistemisch-referentiellen Geltungsanspruch. Grundsätzlich stehen narrative Kausalerklärungen im Gegensatz zu deterministischen oder statistischen, letztlich deduktiv-nomologischen Erklärungen, wie sie für die Naturwissenschaften angenommen werden. Welche Faktoren den Absturz der Boeing 737 Max verursacht haben, mag im Detail strittig sein, ist aber ungeachtet, ja vielleicht sogar gerade wegen der komplexen kausalen Gemengelage durchaus narrativ objektivierbar.

Erzählungen können ein Geschehen jedoch nicht nur erklären, indem sie Ereignisse kausal miteinander verknüpfen, sondern auch dadurch, dass sie eine komplexe Menge von Ereignissen insgesamt zu einer bedeutungsstiftenden Gesamtgestalt konfigurieren. Hayden White bezeichnet ein solches Verfahren bekanntlich als ,emplotment':

\footnotetext{
When the reader recognizes the story being told as a specific kind of story - for example, as an epic, romance, tragedy, comedy, or farce -, he can be said to have comprehended the meaning produced by the discourse. This comprehension is nothing other than the recognition of the form of the narrative. (White 1987, 43)
}

Ein Geschehen zu verstehen, heißt für White, es als Beispielfall eines allgemeinen Geschichtentyps („kind of story“) zu erfassen und damit unter ein Handlungsschema (,plot‘) zu subsumieren. Kohärenz entsteht hier nicht so sehr durch die Annahme plausibler Ursache-Wirkungs-Zusammenhänge, sondern durch die Zuweisung eines übergreifenden Schemas. Dieses konfiguriert und synthetisiert disparate Geschehenselemente zu einem Ganzen. Die Motivation des Geschehens erfolgt hier vom Handlungsschema her. Aus dieser Perspektive geschieht ein Ereignis nicht, weil es durch ein vorheriges verursacht wurde, sondern damit die 
Handlung ihr Schema erfüllt. Die erzählte Geschichte erhält so eine übersummative Qualität: Sie bedeutet mehr als die Summe ihrer Teile. Während die kausalnarrative Erklärung eine singuläre Ereignisfolge additiv als in ihren Einzelteilen typisierbaren, aber insgesamt singulären Ursache-Wirkungs-Zusammenhang verständlich macht, erfasst die konfigurative Erklärung ein individuelles Geschehen insgesamt als Beispielfall eines allgemeinen Typus. Die kognitive Funktion narrativer Konfigurationen besteht nicht darin, eine Serie von Ereignissen darzustellen, sondern vielmehr darin, diese als ein prägnantes Ganzes verstehbar zu machen. Solche konfigurativen Geschehenserklärungen besitzen eine hoch suggestive Überzeugungskraft, weil sie die Diversität zwischen Einzelereignissen überspielen und so kognitive Dissonanzen vermeiden, weil sie unübersichtliche kausale Gemengelagen stark vereinfachen und nicht zuletzt, weil sie ein eigentlich kontingentes Geschehen an vorbewusste kulturelle Grundüberzeugungen anschließen.

Der Absturz der indonesischen Lion Air-Boeing 737 Max könnte beispielsweise, je nach Adressatenkreis und Wirkungsabsicht, verschwörungstheoretisch als tragisches Exempel konfiguriert werden, demzufolge ärmere Länder zum Opfer der Profitgier verantwortungsloser westlicher Hightec-Unternehmen werden, oder auch als Farce, die einmal mehr die unüberwindbare technische Rückständigkeit und organisatorische Fahrlässigkeit der Dritten Welt bestätigt.

\section{Erzählen und Wahrheit}

Wie stehen kausale und konfigurative Erklärungen von Geschehen zueinander? Narrativ-kausales Erklären zielt auf ein prozessuales Erfassen der einzelnen Episoden eines Geschehens in ihrer kausalen Verkettung. Narratives Konfigurieren ist schemabezogen und erfasst das Geschehen als Ganzes. Beide Erklärungstypen verleihen Ereignisketten eine über deren bloße chronologische Ordnung hinausgehende Kohärenz. Im Alltagsgebrauch stehen sie aber interessanterweise weder in einem alternativen noch in einem komplementären Verhältnis zueinander, sondern werden auf eine elastische, unsystematische Weise miteinander vermischt: Ein und dasselbe Geschehen kann mithilfe einer Erzählung kausal erklärt und zugleich durch Subsumption unter ein übergreifendes narratives Schema konfigurativ verstanden werden. Die beiden narrativen Kohärenztypen sind aber auch nicht notwendigerweise kongruent oder koextensiv: Wir können ein Geschehen konfigurieren, ohne es lückenlos kausal zu erklären. Andererseits sind wir in der Lage, ein Geschehen kausal zu erklären, ohne es konfigurativ zu prägen. 
Eine vorurteilsbelastete Konfiguration der Gemengelage technischer und organisatorischer Pannen vor dem Absturz der Lion Air-Boeing 737 Max als Tragödie oder als Farce kann Kausalerklärungen wennschon nicht komplett ignorieren, so doch vernachlässigen, ohne an Stimmigkeit und Überzeugungskraft einzubüßen. Wer der überforderten thailändischen Fluggesellschaft die Schuld für den Absturz geben möchte, wird andere Faktoren in den Vordergrund stellen als derjenige, der die von Geldgier getriebenen Einsparungen des Flugzeugherstellers Boeing belegen will. Andererseits kann eine genaue kausale Rekonstruktion des Boeing-Absturzes die Einseitigkeit und Irrationalität einer verschwörungstheoretischen Interpretation bloßstellen, ohne selbst ein übergreifendes Handlungs- und Deutungsschema anbieten zu müssen.

Narrative Geschehenserklärungen treten in zwei Formen, als kausale und als konfigurative auf. Kausale Erklärungen erheben einen referentiellen Wahrheitsanspruch. Die Wahrheit einer komplexen Kausalerklärung ergibt sich aus der Konjunktion und Gewichtung ihrer einzelnen Ereigniserklärungen. Dagegen zielt eine konfigurative Geschehenserklärung übersummativ auf die narrative Gesamtstruktur. Der Geltungsanspruch einer konfigurativen Geschehenserklärung unterliegt eher Kriterien wie ,Bündigkeit‘, ,Überzeugungskraft‘ oder ,Sinnhaftigkeit‘. Handlungsschemata oder Plot-Typen weisen einem Geschehen eine Sinndimension zu, die nicht in einem referentiellen Sinn wahrheitsfähig ist. Ein und dasselbe anekdotische Material kann in unterschiedlichen Plots organisiert werden.

Der angeblich unvermeidlich rhetorische Charakter von Erzählungen schränkt nur den Wahrheitsanspruch einer Form narrativer Erklärungen ein, nämlich die konfigurative. Kausale narrative Erklärungen hingegen sind nicht mehr, aber auch nicht weniger wahrheitsfähig als empirische Aussagen über unsere Wirklichkeit überhaupt. Konfigurative Erklärungen bemühen sich um Suggestion, kausale um Explikation.

\section{Literaturverzeichnis}

Biegon, Dominika, und Frank Nullmeier. „Narrationen über Narrationen. Stellenwert und Methodologie der Narrationsanalyse“. Politische Narrative. Hg. Frank Gadinger u. a. Wiesbaden: Springer, 2014. 39-65.

D’Ancona, Matthew. Post Truth. The new war on truth and how to fight back. London: Ebury Press, 2017.

Danto, Arthur C. Narration and Knowledge (including the integral text of ,Analytical Philosophy of History'). New York: Columbia UP, 1985. 
Haussmann, Thomas. Erklären und Verstehen. Zur Theorie und Pragmatik der Geschichtswissenschaft. Frankfurt a. M.: Suhrkamp, 1991.

Hendricks, Vincent, und Mads Vestergaard. „Verlorene Wirklichkeit? An der Schwelle zur postfaktischen Demokratie“. Aus Politik und Zeitgeschichte 67.13: Wahrheit (2017): 4-10.

Hennig, Hans C. „Erklären - Verstehen - Erzählen. Die wissenschaftstheoretische Analyse der Historiographie“. Theorien der Geschichtswissenschaft. Hg. Jörn Rüsen und Hans Süssmuth. Düsseldorf: Schwann, 1980: 60-78.

Klein, Christian, und Matías Martínez (Hg.): Wirklichkeitserzählungen. Felder, Formen und Funktionen nicht-literarischen Erzählens. Stuttgart/Weimar: Metzler, 2009.

Komite Nasional Keselamatan Transportasi (Republic of Indonesia): Aircraft Accident Investigation Report. Final KNKT.18.10.35.04. Jakarta 2019.

http://knkt.dephub.go.id/knkt/ntsc_aviation/baru/2018\%20-\%20035\%20-\%20PKLQP\%20Final\%20Report.pdf (7. März 2020)

Lorenz, Chris. Konstruktion der Vergangenheit. Eine Einführung in die Geschichtstheorie. Köln: Böhlau 1997.

Mackie, John L. The Cement of the Universe. A Study of Causation [1974]. Oxford: Oxford UP, 2002.

Martínez, Matías. „Erklären“. Erzählen. Ein interdisziplinäres Handbuch. Hg. Matías Martínez. Stuttgart: Metzler, 2017: 250-257.

N. N.: „,Neun Faktoren“ verursachten Absturz der Boeing 737 von Lion Air. Offizieller Abschlussbericht“. Frankfurter Allgemeine Zeitung, 25.10.2019.

Ockenfels, Axel, und Wilhelm Hofmann. „Warum wir glauben, was wir glauben“. Vom Umgang mit Fakten. Antworten aus Natur-, Sozial- und Geisteswissenschaften. Hg. Günter Blamberger u. a. München: Fink, 2018: 79-89.

Volbert, Renate, und Nina Heering. „Beglaubigen“. Erzählen. Ein interdisziplinäres Handbuch. Hg. Matías Martínez. Stuttgart: Metzler, 2017: 224-231.

White, Hayden: „The Question of Narrative in Contemporary Historical Theory“. In: Hayden White: The Content of the Form. Narrative Discourse and Historical Representation. Baltimore: Johns Hopkins UP, 1987: 26-57. 